\title{
1,1'-(Ethane-1,2-diyl)dipyridinium bistribromide (EDPBT) as a recyclable catalyst for acylation
}

\author{
Sarala Naik, Veerababurao Kavala^, Rangam Gopinath^^and Bhisma K. Patel^* \\ Department of Chemistry, Indian Institute of Technology, Guwahati-781 039, India. \\ E-mail: patel@iitg.ernet.in
}

\begin{abstract}
1,1'-(Ethane-1,2-diyl)dipyridinium bistribromide (EDPBT) catalyzes the acylation of structurally diverse alcohols, amines, thiols, and phenols with a variety of aliphatic and aromatic anhydrides. Steric factors in substrates as well as anhydrides and solvent play significant role during the formation of acylates. Chemoselective mono acetylation of symmetrical diols, primary hydroxy group over secondary and phenolic group and amines over phenols has been achieved. The compatibility of the protocol has been shown by the survival of different acid sensitive functionalities under the present reaction condition. The solvent, acetone, reacts with EDPBT giving bromoacetone and $\mathrm{HBr}$, thus suppressing the bromination of substrates otherwise amenable to bromination. The reagent EDPBT being devoid of phase transfer property and owing to the high solubility of its precursor 1,1'-(ethane-1,2-diyl)dipyridinium dibromide (EDPDB) in water, it was possible to isolate pure acylates by an aqueous work-up circumventing the need for further purification. The process is superior owing to the recyclability of the reagent. The spent reagent can be recovered, regenerated, and reused without any significant loss.
\end{abstract}

Keywords: Catalysis, alcohol, ester, amide, acylation, chemoselective, 1,1'-(ethane-1,2diyl)dipyridinium bistribromide

\section{Introduction}

The corrosive and toxic molecular bromine has recently been replaced by solid organic ammonium tribromides, because of the ease in their storage, transport, handling, and maintenance of desired stoichiometry. ${ }^{1-7}$ These are unique reagents and have found diverse applications in recent years. ${ }^{1-8}$ Their exploration as a source of anhydrous $\mathrm{HBr}$ makes the use of tribromides an attractive alternative to conventional protic and Lewis acids. ${ }^{8}$ Notably, the acidity of the reaction medium using tribromides can be tuned by changing the polarity of the organic

\footnotetext{
All authors have contributed equally
} 
medium. ${ }^{8 \mathrm{e}}$ To overcome the problem of recyclability, as is the case with most organic ammonium tribromides, we have recently reported a new reagent, 1,1'-(ethane-1,2-diyl)dipyridinium bistribromide (EDPBT) containing two tribromide units per molecule. ${ }^{9}$ Unlike other tribromides, this reagent is devoid of phase-transfer property and can be recovered in significant amounts as its precursor 1,1'-(ethane-1,2-diyl)dipyridinium dibromide (EDPDB) is highly water soluble. Taking into account the potential uses of tetrabutylammonium tribromide (TBATB) for different organic functional group transformations and the efficiency and recyclability of EDPBT, make further exploration of the reagent to other functional group transformations useful and viable.

Acylation of protic nucleophiles such as alcohols, amines, and thiols is an important and commonly used reaction in organic chemistry. The resulting esters, amides and thioesters serve as important functional components and / or intermediates in synthetic chemistry and biology. ${ }^{10-12}$ In general acid anhydrides are employed as the acyl sources because of their ready availability and stability. Traditionally, bases such as triethylamine, pyridine, 4(dimethylamino)pyridine (DMAP) and 4-pyrrolidinopyridine (PPY) and tributylphosphine $\left(\mathrm{Bu}_{3} \mathrm{P}\right)$ are employed. ${ }^{13-16}$ Because of their toxicity, flammability and unpleasant odors these bases are less attractive. ${ }^{13}$ Acylation under acid catalyzed conditions has been reported with several reagents. ${ }^{17,18}$ Metal triflates ${ }^{19-29}$ and perchlorates ${ }^{30-33}$ have been used for the purpose owing to their acidic nature. Other reagents / catalysts employed are $\mathrm{TMSCl},{ }^{34} \mathrm{HClO}_{4}-\mathrm{SiO}_{2}{ }^{35}$ $\mathrm{Sc}\left(\mathrm{NTf}_{2}\right)_{3},{ }^{36}$ Nafion-H, ${ }^{37}$ Yttria-Zirconia, ${ }^{38}$ distannoxane ${ }^{15,39}$ heteropoly acid, ${ }^{40} \mathrm{MeSO}_{3} \mathrm{H} / \mathrm{Al}_{2} \mathrm{O}_{3}{ }^{41}$ solid surface- $\mathrm{Al}_{2} \mathrm{O}_{3},{ }^{42}$ and oxomolybdenum species. ${ }^{43}$ Although metal triflates, perchlorates and other acidic catalysts are effective for the acylation reaction, their use is limited due to explosive nature of metal perchlorates and strong acidic character of triflates which results in side reactions. The drawbacks associated with some of the procedures reported in literature are arduous preparation of catalysts, difficulties in work-up and isolation, the need for an inert atmosphere, harsh reaction conditions, expensive reagents, low yields, longer reaction times, dry solvents and incompatibility with other protecting groups. In this context search for achieving general nucleophilic acyl substitution of anhydrides in a catalytic, mild fashion with integrity of existing acid and base sensitive functionalities remains in great demand. Though a plethora of reagents and procedures for acylation have been documented in the literature, need to find an efficient and mild acylation reaction still remains. Herein, we reveal a new, mild procedure for preparation of various esters, amides and thioesters with a variety of aliphatic and aromatic anhydrides in the presence of catalytic quantity of EDPBT.

\section{Results and Discussion}

Initially 3-phenyl propanol 3 (5 mmol) was taken as the model substrate for acetylation and was reacted with acetic anhydride $(6.25 \mathrm{mmol})$ in a donor solvent such as acetonitrile $(10 \mathrm{~mL})$ in the presence of catalytic quantity of EDPBT $(0.25 \mathrm{mmol})$. Progress of the reaction was monitored using thin layer chromatography, which showed complete conversion of the alcohol to its acetate 
within $35 \mathrm{~min}$. Earlier we have reported that the acidity of the reaction medium employing tribromides can be tuned by changing the polarity of the solvent. ${ }^{8}$ So using this fact; the reaction was performed in different solvents such as toluene, methylene chloride, chloroform and acetone. When 3-phenyl propanol 3 was reacted with acetic anhydride in the presence of EDPBT in above solvents separately, it was observed that the reaction proceeded much faster in acetone compared to other solvents. This could be due to the reaction of acetone with EDPBT forming bromoacetone and thereby generating anhydrous $\mathrm{HBr}$ in situ, which catalyzes the reaction. In a control experiment treatment of the reaction mixture with a catalytic quantity of bromine and aq. $\mathrm{HBr}$ instead of EDPBT in acetone yielded $95 \%$ and $88 \%$ of acylated product respectively within 10 minutes. The summary of the solvent dependent study is shown in Table 1 . This result prompted us to use acetone as the reaction medium.

Table 1. Solvent dependent acylation of 3-phenyl propanol (3)

\begin{tabular}{llll}
\hline Substrate & Solvent & Time/min & Yield (\%) ${ }^{[\mathrm{a}]}$ \\
\hline $\mathrm{PhCH}_{2} \mathrm{CH}_{2} \mathrm{CH}_{2} \mathrm{OH}(\mathbf{3})$ & Toluene & 90 & 88 \\
& $\mathrm{CH}_{2} \mathrm{Cl}_{2}$ & 60 & 91 \\
& $\mathrm{CH}_{3} \mathrm{CN}$ & 35 & 93 \\
& $\mathrm{CH}_{3} \mathrm{COCH}_{3}$ & 05 & 95 \\
$\mathrm{PhCH}\left(\mathrm{CH}_{3}\right) \mathrm{OH}(\mathbf{1 6})$ & Toluene & 120 & 80 \\
& $\mathrm{CH}_{2} \mathrm{Cl}_{2}$ & 75 & 86 \\
& $\mathrm{CH}_{3} \mathrm{CN}$ & 50 & 92 \\
& $\mathrm{CH}_{3} \mathrm{COCH}_{3}$ & 15 & 93 \\
\hline
\end{tabular}

${ }^{\mathrm{a}} \mathrm{GC}$ yield

The reagent and the methodology are superior with respect to other conventional reagents as it results in a highly efficient acetylation of 3-phenyl propanol 3 (Table 2). Summary of the acetylation using 0.05 equivalents of different reagents employing acetone as the solvent is shown in Table 2. It may be noted that the yields reported in Table 2 are much lower than those has been reported in the literature, a possible reason could be the use of different amounts of catalyst, acetic anhydride and solvent system.

Under the present optimized reaction condition, diverse arrays of alcohols were converted to their respective acetates (Table 3). Aliphatic primary alcohols 1-3 were transformed to their corresponding acetates in excellent yields in a short time. Benzylic alcohols with deactivated substituents in the aromatic ring 4-5 were also acetylated efficiently. By employing this reagent we carried out acetylation of hindered and deactivated benzylic alcohol $\mathbf{6}$ in excellent yield but with a longer reaction time $24 \mathrm{~h}$. However, other hindered alcohols 7-8 underwent acetylation smoothly giving product in excellent yield. Primary aliphatic diols 9-12 were diacetylated completely with acetic anhydride (2.5 equiv.). When pentaerythritol 13, a substrate containing four symmetrical hydroxyl groups, was reacted with acetic anhydride (5 equiv.) it gave a 
diisopropylidine derivative 13"a along with the isopropylidine diacetate 13'a in the ratio (30:70). It is worth noting that a change of solvent from acetone to acetonitrile resulted in the formation of pentaerythritol tetraacetate 13a as the sole product in nearly quantitative yield $(96 \%)$, Scheme 1 .

Table 2. Acetylation of 3-phenyl propanol 3 in acetone using 0.05 equiv. of the reagents and acetic anhydride (1.25 equiv.)

\begin{tabular}{llll}
\hline Substrate & Reagents used & Time/h & Yield (\%) ${ }^{\mathrm{a}}$ \\
\hline & $\mathrm{ZnCl}_{2}$ & 24 & 30 \\
& $\mathrm{CoCl}_{2}$ & 24 & 35 \\
& $\mathrm{MgBr}_{2}$ & 24 & 40 \\
& $\mathrm{RuCl}_{3}$ & 24 & 70 \\
& $\mathrm{NBS}$ & 8 & 92 \\
$\mathrm{PhCH}_{2} \mathrm{CH}_{2} \mathrm{CH}_{2} \mathrm{OH}(3)$ & $\mathrm{Cu}(\mathrm{OTf})_{2}$ & 24 & 55 \\
& $\mathrm{LiClO}_{4}$ & 24 & 60 \\
& $\mathrm{HClO}_{4}-\mathrm{SiO}_{2}$, & 24 & 30 \\
& EDPBT & 0.25 & 98 \\
\hline
\end{tabular}

${ }^{\mathrm{a}} \mathrm{GC}$ yield

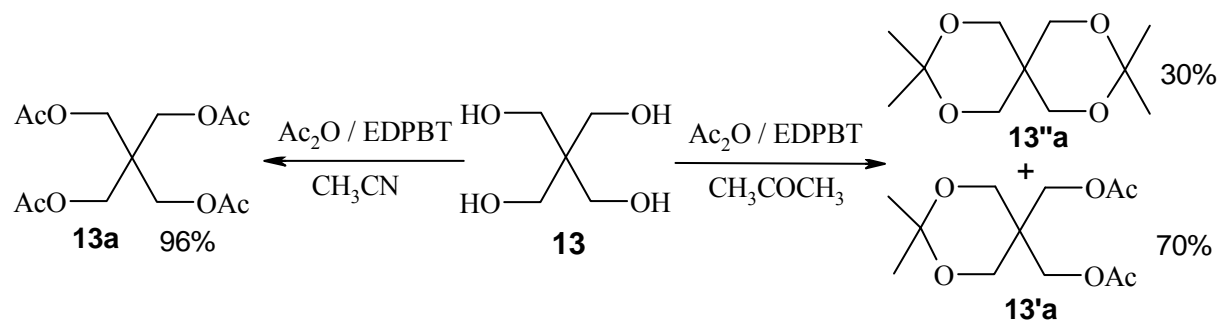

Scheme 1. Acetylation and isopropylidination of pentaerythritol 13.

Secondary alicyclic alcohol such as cyclohexanol 14 was converted to its acetate in good yield. It was gratifying to observe that menthol 15 was completely converted to menthyl acetate 15a with 1.25 equiv of $\mathrm{Ac}_{2} \mathrm{O}$ within $10 \mathrm{~min}$, an earlier work has reported that it was acetylated in $1.5 \mathrm{~h}$ at $0^{\circ} \mathrm{C}$ with 5 equivalents of $\mathrm{Ac}_{2} \mathrm{O}$ in the presence of $1 \mathrm{~mol} \%$ of $\mathrm{Bi}(\mathrm{OTf})_{3}{ }^{21} \mathrm{Benzylic}$ secondary alcohols such as 16-19 underwent acetylation smoothly but with a slightly longer reaction time as compared to their primary analogues. However, the scope of acetylation is somewhat limited for hindered aromatic secondary alcohol 9-fluorenyl alcohol 20, which took 5 $\mathrm{h}$ for complete conversion under reflux. The results obtained have been summarized in Table 3 . 
Table 3. Acetylation of alcohols with $\mathrm{Ac}_{2} \mathrm{O}$ catalyzed by EDPBT in acetone

\begin{tabular}{|c|c|c|c|}
\hline Substrate & Product & Time/min & Yield $(\%)^{b}$ \\
\hline $\mathrm{CH}_{3}\left(\mathrm{CH}_{2}\right)_{8} \mathrm{CH}_{2} \mathrm{OH}(\mathbf{1})$ & $\mathrm{CH}_{3}\left(\mathrm{CH}_{2}\right)_{8} \mathrm{CH}_{2} \mathrm{OAc}(\mathbf{1 a})$ & 5 & 92 \\
\hline $\mathrm{CH}_{3}\left(\mathrm{CH}_{2}\right)_{10} \mathrm{CH}_{2} \mathrm{OH}(\mathbf{2})$ & $\mathrm{CH}_{3}\left(\mathrm{CH}_{2}\right)_{10} \mathrm{CH}_{2} \mathrm{OAc}(\mathbf{2 a})$ & 5 & 93 \\
\hline $\mathrm{Ph}\left(\mathrm{CH}_{2}\right)_{2} \mathrm{CH}_{2} \mathrm{OH}(\mathbf{3})$ & $\mathrm{Ph}\left(\mathrm{CH}_{2}\right)_{2} \mathrm{CH}_{2} \mathrm{OAc}(\mathbf{3 a})$ & 5 & 95 \\
\hline $3-\mathrm{NO}_{2}-\mathrm{C}_{6} \mathrm{H}_{4}-\mathrm{CH}_{2} \mathrm{OH}(4)$ & $3-\mathrm{NO}_{2}-\mathrm{C}_{6} \mathrm{H}_{4}-\mathrm{CH}_{2} \mathrm{OAc}(\mathbf{4 a})$ & 15 & 90 \\
\hline 4-Cl- $\mathrm{C}_{6} \mathrm{H}_{4}-\mathrm{CH}_{2} \mathrm{OH}(\mathbf{5})$ & $4-\mathrm{Cl}-\mathrm{C}_{6} \mathrm{H}_{4}-\mathrm{CH}_{2} \mathrm{OAc}(\mathbf{5 a})$ & 10 & 92 \\
\hline 2-Cl-6- $\mathrm{NO}_{2}-\mathrm{C}_{6} \mathrm{H}_{3}-\mathrm{CH}_{2} \mathrm{OH}(\mathbf{6})$ & 2-Cl-6- $\mathrm{NO}_{2}-\mathrm{C}_{6} \mathrm{H}_{3}-\mathrm{CH}_{2} \mathrm{OH}(\mathbf{6 a})$ & $24 \mathrm{~h}$ & 80 \\
\hline 9-Flurenyl- $\mathrm{CH}_{2} \mathrm{OH}(7)$ & 9-Flurenyl- $\mathrm{CH}_{2} \mathrm{OAc}(\mathbf{7 a})$ & 10 & 95 \\
\hline$\left(\mathrm{PhCH}_{2}\right)_{2} \mathrm{~N}\left(\mathrm{CH}_{2}\right)_{2} \mathrm{OH}(\mathbf{8})$ & $\left(\mathrm{PhCH}_{2}\right)_{2} \mathrm{~N}\left(\mathrm{CH}_{2}\right)_{2} \mathrm{OAc}(\mathbf{8 a})$ & 10 & 92 \\
\hline $\mathrm{HO}\left(\mathrm{CH}_{2}\right)_{5} \mathrm{OH}(\mathbf{9})$ & $\mathrm{AcO}\left(\mathrm{CH}_{2}\right)_{5} \mathrm{OAc}(\mathbf{9 a})$ & 10 & $89^{c}$ \\
\hline $\mathrm{HOCH}_{2} \mathrm{CH}_{2} \mathrm{OCH}_{2} \mathrm{CH}_{2} \mathrm{OH}(\mathbf{1 0})$ & $\mathrm{AcOCH}_{2} \mathrm{CH}_{2} \mathrm{OCH}_{2} \mathrm{CH}_{2} \mathrm{OAc}(\mathbf{1 0 a})$ & 10 & $90^{\mathrm{c}}$ \\
\hline $\mathrm{HOCH}_{2} \mathrm{C}\left(\mathrm{CH}_{3}\right)_{2} \mathrm{CH}_{2} \mathrm{OH}$ (11) & $\mathrm{AcOCH}_{2} \mathrm{C}\left(\mathrm{CH}_{3}\right)_{2} \mathrm{CH}_{2} \mathrm{OAc}$ (11a) & 10 & $90^{\mathrm{c}}$ \\
\hline 3-Chloro-1,2-propane diol (12) & 1,2-Diacetoxy-3-chloro propane (12a) & 10 & $90^{\mathrm{c}}$ \\
\hline $\mathrm{C}\left(\mathrm{CH}_{2} \mathrm{OH}\right)_{4}(\mathbf{1 3})$ & $\left(\mathrm{AcOCH}_{2}\right)_{2} \mathrm{C}\left[\left(\mathrm{CH}_{2} \mathrm{O}\right)_{2} \mathrm{C}\left(\mathrm{CH}_{3}\right)_{2}\right]\left(\mathbf{1 3}^{\prime} \mathbf{a}\right)$ & 60 & $70^{\mathrm{d}}$ \\
\hline $\mathrm{C}\left(\mathrm{CH}_{2} \mathrm{OH}\right)_{4}(\mathbf{1 3})$ & $\mathrm{C}\left(\mathrm{CH}_{2} \mathrm{OAc}\right)_{4}(\mathbf{1 3 a})$ & $5 \mathrm{~h}$ & $96^{\mathrm{e}}$ \\
\hline Cyclohexanol (14) & Cyclohexyl acetate (14a) & 10 & 85 \\
\hline Menthol (15) & Menthyl acetate (15a) & 10 & 87 \\
\hline $\mathrm{C}_{6} \mathrm{H}_{5}-\mathrm{CH}\left(\mathrm{CH}_{3}\right) \mathrm{OH}(\mathbf{1 6})$ & $\mathrm{C}_{6} \mathrm{H}_{5}-\mathrm{CH}\left(\mathrm{CH}_{3}\right) \mathrm{OAc}(\mathbf{1 6 a})$ & 15 & 92 \\
\hline $3-\mathrm{NO}_{2}-\mathrm{C}_{6} \mathrm{H}_{4}-\mathrm{CH}\left(\mathrm{CH}_{3}\right) \mathrm{OH}(\mathbf{1 7})$ & $3-\mathrm{NO}_{2}-\mathrm{C}_{6} \mathrm{H}_{4}-\mathrm{CH}\left(\mathrm{CH}_{3}\right) \mathrm{OAc}(\mathbf{1 7 a})$ & 35 & 84 \\
\hline $4-\mathrm{Cl}-\mathrm{C}_{6} \mathrm{H}_{4}-\mathrm{CH}\left(\mathrm{CH}_{3}\right) \mathrm{OH}(\mathbf{1 8})$ & 4-Cl- $\mathrm{C}_{6} \mathrm{H}_{4}-\mathrm{CH}\left(\mathrm{CH}_{3}\right) \mathrm{OAc}(\mathbf{1 8 a})$ & 35 & 89 \\
\hline$\left(\mathrm{C}_{6} \mathrm{H}_{5}\right)_{2} \mathrm{CHOH}(\mathbf{1 9})$ & $\left(\mathrm{C}_{6} \mathrm{H}_{5}\right)_{2} \mathrm{CHOAc}(\mathbf{1 9 a})$ & 30 & 89 \\
\hline 9-Fluorenyl-OH (20) & 9-Fluorenyl OAc (20a) & $5 \mathrm{~h}$ & $79^{\mathrm{f}}$ \\
\hline
\end{tabular}

${ }^{a}$ Reactions were monitored by TLC. ${ }^{b}$ Isolated yield. ${ }^{c} 2.5$ equivs. of $\mathrm{Ac}_{2} \mathrm{O}$ were used. ${ }^{\mathrm{d}} 5$ equivs. of $\mathrm{Ac}_{2} \mathrm{O}$ were used in acetone. ${ }^{\mathrm{e}} 5$ equivs. of $\mathrm{Ac}_{2} \mathrm{O}$ were used in $\mathrm{CH}_{3} \mathrm{CN}$. ${ }^{\mathrm{f}} \mathrm{Reflux}$ condition.

This methodology was also successfully applied to a representative variety of functionalized alcohols (Table 4). Diacylated product was obtained without affecting the multiple bonds in substrates, but-2-ene-1,4-diol 21 and but-2-yne-1,4-diol 22 with acetic anhydride (2.5 equiv.). Cholesterol 23 took comparatively longer reaction time for the transformation. A substrate containing two hydroxyls and a NHBoc functionality $\mathbf{2 6}$ gave monoacylated product without affecting the NHBoc group when reacted with acetic anhydride (1.25 equiv.). The present method is also effective for acylation of $\alpha, \beta$-unsaturated alcohol such as cinnamyl 24. Conversion of 4-allyloxy benzyl alcohol 25 to its acetate also occurred smoothly without affecting the double bond. Moreover, substrates bearing acid sensitive groups such as NHBoc 26, OMe 27, THP 28, and TBS ether 31, and base sensitive groups such as benzoate 2930 remained intact under the described reaction condition revealing the functional group compatibility. The pKas of $\mathrm{HBr}, \mathrm{HClO}_{4}$ and triflic acid respectively are $-9,-10$, and -13 showing that triflic and perchloric acids have stronger acidic character compared to $\mathrm{HBr}$. It may 
be noted that strong Lewis acid character of metal triflates makes them unsuitable for acidsensitive substrates. In scandium triflate catalyzed acetylation, rearranged products of allylic alcohols have been observed. ${ }^{24 d}$ Results obtained for differential functionalized alcohols have been summarized in Table 4 .

Table 4. EDPBT catalyzed acetylation ${ }^{\mathrm{a}}$ of functionalized alcohols in acetone

\begin{tabular}{|c|c|c|c|}
\hline Substrate & Product & Time/min & Yield $(\%)^{\mathrm{b}}$ \\
\hline $\mathrm{HOCH}_{2} \mathrm{CH}=\mathrm{CHCH}_{2} \mathrm{OH}(\mathbf{2 1})$ & $\mathrm{AcOCH}_{2} \mathrm{CH}=\mathrm{CHCH}_{2} \mathrm{OAc}$ (21a) & 60 & $84^{\mathrm{c}}$ \\
\hline 2-Butyne-1,4-diol (22) & 1,4-Diacetoxy-2-butyne (22a) & 75 & $86^{\mathrm{c}}$ \\
\hline Cholesterol (23) & Cholesteryl acetate (23a) & $8 \mathrm{~h}$ & 90 \\
\hline $\mathrm{PhCH}=\mathrm{CH}-\mathrm{CH}_{2} \mathrm{OH}(\mathbf{2 4})$ & $\mathrm{PhCH}=\mathrm{CH}-\mathrm{CH}_{2} \mathrm{OAc}(\mathbf{2 4 a})$ & 05 & 89 \\
\hline 4-Allyloxy- $\mathrm{C}_{6} \mathrm{H}_{4}-\mathrm{CH}_{2} \mathrm{OH}(\mathbf{2 5})$ & 4-Alloylloxy- $\mathrm{C}_{6} \mathrm{H}_{4}-\mathrm{CH}_{2} \mathrm{OAc}(\mathbf{2 5 a})$ & 20 & 90 \\
\hline $\mathrm{OHCH}_{2} \mathrm{C}\left(\mathrm{CH}_{3}, \mathrm{NH}(\mathrm{Boc}) \mathrm{CH}_{2} \mathrm{OH}(\mathbf{2 6})\right.$ & $\mathrm{AcOCH}_{2} \mathrm{C}\left(\mathrm{CH}_{3}, \mathrm{NH}(\mathrm{Boc}) \mathrm{CH}_{2} \mathrm{OH}\right.$ (26a) & 90 & 70 \\
\hline 4-OMe- $\mathrm{C}_{6} \mathrm{H}_{4}-\mathrm{CH}_{2} \mathrm{OH}(27)$ & $4-\mathrm{OMe}-\mathrm{C}_{6} \mathrm{H}_{4}-\mathrm{CH}_{2} \mathrm{OAc}(\mathbf{2 7} \mathbf{a})$ & 10 & 92 \\
\hline $\mathrm{THPO}\left(\mathrm{CH}_{2}\right)_{5} \mathrm{CH}_{2} \mathrm{OH}(\mathbf{2 8})$ & $\mathrm{THPO}\left(\mathrm{CH}_{2}\right)_{5} \mathrm{CH}_{2} \mathrm{OAc}(\mathbf{2 8 a})$ & 10 & 87 \\
\hline $\mathrm{C}_{6} \mathrm{H}_{5}-\mathrm{COOCH}_{2} \mathrm{CH}_{2} \mathrm{OH}(\mathbf{2 9})$ & $\mathrm{C}_{6} \mathrm{H}_{5}-\mathrm{COOCH}_{2} \mathrm{CH}_{2} \mathrm{OAc}$ (29a) & 30 & 92 \\
\hline $\mathrm{C}_{6} \mathrm{H}_{5}-\mathrm{COO}\left(\mathrm{CH}_{2}\right)_{2} \mathrm{OCH}_{2} \mathrm{CH}_{2} \mathrm{OH}(\mathbf{3 0})$ & $\mathrm{C}_{6} \mathrm{H}_{5-} \mathrm{COO}\left(\mathrm{CH}_{2}\right)_{2} \mathrm{OCH}_{2} \mathrm{CH}_{2} \mathrm{OAc}(\mathbf{3 0 a})$ & 40 & 94 \\
\hline 4-OTBDMs- $\mathrm{C}_{6} \mathrm{H}_{4}-\mathrm{CH}_{2} \mathrm{CH}_{2} \mathrm{OH}(\mathbf{3 1})$ & 4-OTBDMs- $\mathrm{C}_{6} \mathrm{H}_{4}-\mathrm{CH}_{2} \mathrm{CH}_{2} \mathrm{OAc}$ (31a) & 60 & 85 \\
\hline
\end{tabular}

${ }^{\mathrm{a}} \mathrm{Reactions}$ were monitored by TLC. ${ }^{\mathrm{b}}$ Isolated yield. ${ }^{\mathrm{c}} 2.5$ equivs of $\mathrm{Ac}_{2} \mathrm{O}$ were used.

The acetylation of a wide range of structurally varied aliphatic, benzylic, allylic alcohols and phenols highlight the fact that the method is capable of generalization. However, phenols were sluggish under the present reaction condition and took comparatively longer reaction time for completion (Table 5). This result was attributed to the differential nucleophilicities of phenols and aliphatic alcohols under the reaction condition. It is noteworthy to quote that phenols are less nucleophilic than aliphatic alcohols under acidic condition but more nucleophilic under basic condition. ${ }^{2 \mathrm{~b}}$ Phenols containing electron donating groups in the aromatic ring 33-35 were acetylated with ease in comparison to those with electron-withdrawing groups 36-37 (Table 5).

Numerous procedures have been reported in literature for acylation of amines. ${ }^{10,48,49}$ Some of the catalysts that are capable of acylating alcohols, phenols, thiols and amines are $\mathrm{RuCl}_{3},{ }^{18 \mathrm{e}}$ $\mathrm{InCl}_{3}{ }^{18 \mathrm{f}}{ }^{\mathrm{BiOClO}}{ }_{4},{ }^{33}$ heteropoly acid, ${ }^{40}$ solid surface- $\mathrm{Al}_{2} \mathrm{O}_{3},{ }^{42}$ oxomolybdenum species, ${ }^{43}$ $\mathrm{V}(\mathrm{O})(\mathrm{OTf})_{2}{ }^{44}$ The versatility of the described procedure can be observed from its successful application to N- and S-acylation of structurally different amines and thiols. Primary aromatic amines 38-40 and anilines with various substituents 41-44 were converted to their corresponding amides in short time, whereas secondary amine $\mathbf{4 5}$ took hours for completion. This result is not surprising since $\mathrm{N}$-acylation of primary amines has been carried out without the use of any acidic or basic catalyst. ${ }^{50}$ Thioacetal and hemithioacetal were obtained as side products when acylation of dodecanethiol $\mathbf{4 6}$ was carried out in acetone. However, the corresponding thioacetate was obtained by changing the solvent to acetonitrile. The results have been summarized in Table 5 . 
Table 5. Acetylation ${ }^{\mathrm{a}}$ of phenols, amines and thiols with $\mathrm{Ac}_{2} \mathrm{O}$ catalyzed by EDPBT

\begin{tabular}{|c|c|c|c|}
\hline Substrate & Product & Time / min & Yield $(\%)^{\mathrm{b}}$ \\
\hline $\mathrm{C}_{6} \mathrm{H}_{5}-\mathrm{OH}(\mathbf{3 2})$ & $\mathrm{C}_{6} \mathrm{H}_{5}-\mathrm{OAc}$ (32a) & $5 \mathrm{~h}$ & 80 \\
\hline $2-\mathrm{Me}-\mathrm{C}_{6} \mathrm{H}_{4}-\mathrm{OH}(\mathbf{3 3})$ & 2-Me- $\mathrm{C}_{6} \mathrm{H}_{4}-\mathrm{OAc}(\mathbf{3 3 a})$ & $3 \mathrm{~h}$ & 82 \\
\hline $4-\mathrm{OH}-\mathrm{C}_{6} \mathrm{H}_{4}-\mathrm{OH}(\mathbf{3 4})$ & 4-OAc- $\mathrm{C}_{6} \mathrm{H}_{4}-\mathrm{OAc}(\mathbf{3 4 a})$ & $5 \mathrm{~h}$ & 85 \\
\hline $2-\mathrm{OH}-\mathrm{C}_{6} \mathrm{H}_{4}-\mathrm{OH}(\mathbf{3 5})$ & 2-OAc- $\mathrm{C}_{6} \mathrm{H}_{4}-\mathrm{OAc}(\mathbf{3 5 a})$ & $5 \mathrm{~h}$ & 78 \\
\hline 4- $\mathrm{NO}_{2}-\mathrm{C}_{6} \mathrm{H}_{4}-\mathrm{OH}(\mathbf{3 6})$ & 4- $\mathrm{NO}_{2}-\mathrm{C}_{6} \mathrm{H}_{4}-\mathrm{OAc}(\mathbf{3 6 a})$ & $24 \mathrm{~h}$ & 25 \\
\hline $4-\mathrm{CN}-\mathrm{C}_{6} \mathrm{H}_{4}-\mathrm{OH}(37)$ & $4-\mathrm{CN}-\mathrm{C}_{6} \mathrm{H}_{4}-\mathrm{OAc}(\mathbf{3 7 a})$ & $24 \mathrm{~h}$ & 15 \\
\hline $\mathrm{CH}_{3}\left(\mathrm{CH}_{2}\right)_{2} \mathrm{CH}_{2} \mathrm{NH}_{2}(\mathbf{3 8})$ & $\mathrm{CH}_{3}\left(\mathrm{CH}_{2}\right)_{2} \mathrm{CH}_{2} \mathrm{NHAc}$ (38a) & 05 & 80 \\
\hline $\mathrm{C}_{6} \mathrm{H}_{5}-\mathrm{CH}_{2}-\mathrm{NH}_{2}$ (39) & $\mathrm{C}_{6} \mathrm{H}_{5}-\mathrm{CH}_{2}-\mathrm{NHAc}(\mathbf{3 9 a})$ & 05 & 89 \\
\hline $\mathrm{Ph}-\mathrm{CH}\left(\mathrm{CH}_{3}\right) \mathrm{NH}_{2}(\mathbf{4 0})$ & $\mathrm{Ph}-\mathrm{CH}\left(\mathrm{CH}_{3}\right) \mathrm{NHAc}(\mathbf{4 0 a})$ & 05 & 85 \\
\hline $\mathrm{C}_{6} \mathrm{H}_{5} \mathrm{NH}_{2}(\mathbf{4 1})$ & $\mathrm{C}_{6} \mathrm{H}_{5} \mathrm{NHAc}(41 \mathrm{a})$ & 05 & 95 \\
\hline 4-Me- $\mathrm{C}_{6} \mathrm{H}_{4}-\mathrm{NH}_{2}(\mathbf{4 2})$ & 4-Me- $\mathrm{C}_{6} \mathrm{H}_{4}-\mathrm{NHAc}(\mathbf{4 2 a})$ & 05 & 92 \\
\hline $2-\mathrm{F}-\mathrm{C}_{6} \mathrm{H}_{4}-\mathrm{NH}_{2}(43)$ & 2-F-C $\mathrm{H}_{4}-\mathrm{NHAc}(\mathbf{4 3 a})$ & 05 & 90 \\
\hline 4- $\mathrm{NH}_{2}-\mathrm{C}_{6} \mathrm{H}_{4}-\mathrm{NH}_{2}(\mathbf{4 4})$ & 4-NHAc- $\mathrm{C}_{6} \mathrm{H}_{4}-\mathrm{NHAc}(\mathbf{4 4 a})$ & 05 & 92 \\
\hline$\left(\mathrm{C}_{6} \mathrm{H}_{5}\right)_{2} \mathrm{NH}(\mathbf{4 5})$ & $\left(\mathrm{C}_{6} \mathrm{H}_{5}\right)_{2} \mathrm{NAc}(\mathbf{4 5 a})$ & $16 \mathrm{~h}$ & 75 \\
\hline $\mathrm{CH}_{3}\left(\mathrm{CH}_{2}\right)_{10} \mathrm{CH}_{2} \mathrm{SH}(46)$ & $\mathrm{CH}_{3}\left(\mathrm{CH}_{2}\right)_{10} \mathrm{CH}_{2} \mathrm{SAc}(\mathbf{4 6 a})$ & 60 & $70^{\mathrm{c}}$ \\
\hline
\end{tabular}

${ }^{a}$ Reactions were monitored by TLC. ${ }^{b}$ Isolated yield. ${ }^{c} \mathrm{CH}_{3} \mathrm{CN}$ was used as the solvent.

The marginal difference in acylation rate between primary and secondary alcohols, particularly for diols containing both types of hydroxyl yields substantial amount of diacylate. ${ }^{51 a}$ Selective monoacylation of symmetrical as well as unsymmetrical diols with various reagents using symmetrical anhydrides have been reported. ${ }^{15,21 e, 23 b, 25,27,39-42,51}$ High selectivity was obtained for unsymmetrical aliphatic diol possessing both primary and secondary hydroxyl groups such as 1,3-butanediol $\mathbf{4 7}$ when the present method was employed. The primary hydroxyl group was selectively acetylated prior to secondary one with lot wise addition of acetic anhydride (1.2 equiv.) in the presence of EDPBT (0.05 equiv) in acetone. However, lower selectivity was observed for symmetrical primary diols $\mathbf{9}$ and $\mathbf{2 1}$ even with lot wise addition of the anhydride. The ratios of mono and diacetylated product obtained for substrates pentane-1,5diol 9 and but-2-ene-1,4-diol 21 were respectively 65:25 and 75:20. The poor reactivity of phenols with acetic anhydride in presence of EDPBT raised a genuine possibility of selective acylation of aliphatic alcohols over phenols. For substrate $\mathbf{4 8}$ containing both primary and phenolic hydroxyl group, selective monoacetylation occurred at the aliphatic hydroxyl giving exclusively monoacetylated product 48a. Substrate $p$-aminophenol 49 produced the corresponding acetamide; without affecting the phenolic group. Selective N-acetylation is of significant interest for the preparation of the antipyretic and analgesic drug paracetamol 49a. Results obtained have been summarized below in Table 6 . 
Table 6. Selective monoacetylation ${ }^{\mathrm{a}}$ of diols with $\mathrm{Ac}_{2} \mathrm{O}$ catalyzed by EDPBT in acetone

\begin{tabular}{|c|c|c|c|}
\hline Substrate & Product & Time / min & Yield $(\%)^{\mathrm{b}}$ \\
\hline \multirow[t]{2}{*}{$\mathrm{HOCH}_{2}\left(\mathrm{CH}_{2}\right)_{3} \mathrm{CH}_{2} \mathrm{OH}(\mathbf{9})$} & $\mathrm{HOCH}_{2}\left(\mathrm{CH}_{2}\right)_{3} \mathrm{CH}_{2} \mathrm{OAc}(\mathbf{9} \mathbf{a})$ & 05 & 65 \\
\hline & $\mathrm{AcOCH}_{2}\left(\mathrm{CH}_{2}\right)_{3} \mathrm{CH}_{2} \mathrm{OAc}(\mathbf{9 a})$ & & 25 \\
\hline \multirow[t]{2}{*}{$\mathrm{HOCH}_{2}-\mathrm{CH}=\mathrm{CH}-\mathrm{CH}_{2} \mathrm{OH}(\mathbf{2 1})$} & $\mathrm{HOCH}_{2}-\mathrm{CH}=\mathrm{CH}-\mathrm{CH}_{2} \mathrm{OAc}(\mathbf{2 1}$ 'a) & 30 & 75 \\
\hline & $\mathrm{AcOCH}_{2}-\mathrm{CH}=\mathrm{CH}-\mathrm{CH}_{2} \mathrm{OAc}(\mathbf{2 1 a})$ & & 20 \\
\hline \multirow[t]{2}{*}{$\mathrm{HO}\left(\mathrm{CH}_{2}\right)_{2} \mathrm{CHOHCH}_{3}(47)$} & $\mathrm{AcO}\left(\mathrm{CH}_{2}\right)_{2} \mathrm{CH}(\mathrm{OH}) \mathrm{CH}_{3}\left(\mathbf{4} \mathbf{7}^{\prime} \mathbf{a}\right)$ & 10 & 80 \\
\hline & $\mathrm{AcO}\left(\mathrm{CH}_{2}\right)_{2} \mathrm{CH}(\mathrm{OAc}) \mathrm{CH}_{3}(\mathbf{4 7 a})$ & & 15 \\
\hline \multirow[t]{2}{*}{ 4-OH- $\mathrm{C}_{6} \mathrm{H}_{4}-\mathrm{CH}_{2} \mathrm{CH}_{2} \mathrm{OH}(\mathbf{4 8})$} & 4-OH- $\mathrm{C}_{6} \mathrm{H}_{4}-\mathrm{CH}_{2} \mathrm{CH}_{2} \mathrm{OAc}(\mathbf{4 8 a})$ & 10 & 85 \\
\hline & 4-OAc- $\mathrm{C}_{6} \mathrm{H}_{4}-\mathrm{CH}_{2} \mathrm{CH}_{2} \mathrm{OAc}(\mathbf{4 8} \mathbf{a})$ & & 00 \\
\hline \multirow[t]{2}{*}{ 4-OH- $\mathrm{C}_{6} \mathrm{H}_{4}-\mathrm{NH}_{2}(49)$} & 4-OH- $\mathrm{C}_{6} \mathrm{H}_{4}-\mathrm{NHAc}(\mathbf{4 9 a})$ & 05 & 90 \\
\hline & 4-OAc- $\mathrm{C}_{6} \mathrm{H}_{4}-\mathrm{NHAc}(\mathbf{4 9} \mathbf{a})$ & & 00 \\
\hline
\end{tabular}

${ }^{\mathrm{a}}$ Reactions were monitored by TLC. ${ }^{\mathrm{b}}$ Isolated yield

There are few reports of pivaloylation, benzoylation and acylation using other anhydride and alcohols. ${ }^{19,21,24,43}$ It is worth noting that none of the procedures reported in literature has focused on the acylation of alcohols, amines, and thiols with isobutyric anhydride. In order to extend the scope of the methodology, acylation of alcohols, amines, and thiols with other anhydrides was carried out under the identical condition as described using acetic anhydride. A variety of aliphatic and aromatic alcohols $(1,3,16,24)$, amine 41 and thiol 46 were propionylated using propionic anhydride. Chemoselective propionylation of primary alcohol over phenol, and amine over phenol could be observed as demonstrated for substrates 48 and 49 respectively as shown in Table 7 .

Table 7. Propionylation ${ }^{\mathrm{a}}$ of alcohols, thiol and amines with $(\mathrm{EtCO})_{2} \mathrm{O}$ catalyzed by EDPBT

\begin{tabular}{|c|c|c|c|}
\hline Substrate & Product & Time / min & Yield $(\%)^{\mathrm{b}}$ \\
\hline $\mathrm{CH}_{3}\left(\mathrm{CH}_{2}\right)_{8} \mathrm{CH}_{2} \mathrm{OH}(\mathbf{1})$ & $\mathrm{CH}_{3}\left(\mathrm{CH}_{2}\right)_{8} \mathrm{CH}_{2} \mathrm{OCOEt}(\mathbf{1 b})$ & 15 & 88 \\
\hline $\mathrm{Ph}\left(\mathrm{CH}_{2}\right)_{2} \mathrm{CH}_{2} \mathrm{OH}(3)$ & $\mathrm{Ph}-\left(\mathrm{CH}_{2}\right)_{2} \mathrm{CH}_{2} \mathrm{OCOEt}(\mathbf{3 b})$ & 15 & 94 \\
\hline $\mathrm{Ph}-\mathrm{CH}\left(\mathrm{CH}_{3}\right) \mathrm{OH}(\mathbf{1 6})$ & $\mathrm{Ph}-\mathrm{CH}\left(\mathrm{CH}_{3}\right) \mathrm{OCOEt}(\mathbf{1 6 b})$ & 30 & 90 \\
\hline $\mathrm{PhCH}=\mathrm{CHCH}_{2} \mathrm{OH}(\mathbf{2 4})$ & $\mathrm{PhCH}=\mathrm{CHCH}_{2} \mathrm{OCOEt}(\mathbf{2 4 b})$ & 15 & 93 \\
\hline $\mathrm{C}_{6} \mathrm{H}_{5}-\mathrm{NH}_{2}(\mathbf{4 1})$ & $\mathrm{C}_{6} \mathrm{H}_{5}-\mathrm{NHCOEt}(\mathbf{4 1 b})$ & 05 & 95 \\
\hline $\mathrm{CH}_{3}\left(\mathrm{CH}_{2}\right)_{10} \mathrm{CH}_{2} \mathrm{SH}(\mathbf{4 6})$ & $\mathrm{CH}_{3}\left(\mathrm{CH}_{2}\right)_{10} \mathrm{CH}_{2} \mathrm{SCOEt}(\mathbf{4 6 b})$ & 60 & $72^{\mathrm{c}}$ \\
\hline $4-\mathrm{OH}-\mathrm{C}_{6} \mathrm{H}_{4}-\mathrm{CH}_{2} \mathrm{CH}_{2} \mathrm{OH}(\mathbf{4 8})$ & 4-OH- $\mathrm{C}_{6} \mathrm{H}_{4}-\mathrm{CH}_{2} \mathrm{CH}_{2} \mathrm{OCOEt}(\mathbf{4 8 b})$ & 10 & 82 \\
\hline $4-\mathrm{OH}-\mathrm{C}_{6} \mathrm{H}_{4}-\mathrm{NH}_{2}(49)$ & 4-OH- $\mathrm{C}_{6} \mathrm{H}_{4}-\mathrm{NHCOEt}(\mathbf{4 9} \mathbf{b})$ & 10 & 96 \\
\hline
\end{tabular}

${ }^{\mathrm{a}}$ Reactions were monitored by TLC. ${ }^{\mathrm{b}}$ Isolated yield. ${ }^{\mathrm{c}} \mathrm{CH}_{3} \mathrm{CN}$ was used as the solvent

Other anhydrides such as isobutyric and pivalic reacted successfully as shown in Table 8 and 9 respectively. It is needless to mention that chemoselective isobutyrylation and 
pivaloylation of primary alcohol over phenol was observed as demonstrated for substrate 48 (Table 8 and 9).

Table 8. Isobutyrylation ${ }^{\mathrm{a}}$ of alcohols, thiol and amines with (iPrCO) ${ }_{2} \mathrm{O}$ catalyzed by EDPBT

\begin{tabular}{llll}
\hline Substrate & Product & Time/min & Yield (\%) \\
\hline $\mathrm{CH}_{3}-\left(\mathrm{CH}_{2}\right)_{8} \mathrm{CH}_{2} \mathrm{OH}(\mathbf{1})$ & $\mathrm{CH}_{3}-\left(\mathrm{CH}_{2}\right)_{8} \mathrm{CH}_{2} \mathrm{OCO} \operatorname{Pr}(\mathbf{1 c})$ & 20 & 89 \\
$\mathrm{Ph}-\left(\mathrm{CH}_{2}\right)_{2} \mathrm{CH}_{2} \mathrm{OH} \mathrm{(3)}$ & $\mathrm{Ph}-\left(\mathrm{CH}_{2}\right)_{2} \mathrm{CH}_{2} \mathrm{OCO} \operatorname{Pr}(\mathbf{3 c})$ & 20 & 92 \\
$\mathrm{Ph}-\mathrm{CH}\left(\mathrm{CH}_{3}\right) \mathrm{OH}(\mathbf{1 6})$ & $\mathrm{Ph}-\mathrm{CH}\left(\mathrm{CH}_{3}\right) \mathrm{OCO} \operatorname{Pr}(\mathbf{1 6 c})$ & 30 & 88 \\
$\mathrm{PhCH}=\mathrm{CHCH}_{2} \mathrm{OH}(\mathbf{2 4})$ & $\mathrm{PhCH}=\mathrm{CHCH}_{2} \mathrm{OCOiPr}(\mathbf{2 4 c})$ & 15 & 80 \\
$\mathrm{C}_{6} \mathrm{H}_{5}-\mathrm{NH}_{2}(\mathbf{4 1})$ & $\mathrm{C}_{6} \mathrm{H}_{5}-\mathrm{NHCOiPr}^{(41 \mathbf{c})}$ & 05 & 94 \\
$\mathrm{CH}_{3}\left(\mathrm{CH}_{2}\right)_{10} \mathrm{CH}_{2} \mathrm{SH} \mathrm{(46)}$ & $\mathrm{CH}_{3}\left(\mathrm{CH}_{2}\right)_{10} \mathrm{CH}_{2} \mathrm{SCO} \operatorname{Pr}(\mathbf{4 6 c})$ & 60 & $78^{\mathrm{c}}$ \\
$4-\mathrm{OH}-\mathrm{C}_{6} \mathrm{H}_{4}-\mathrm{CH}_{2} \mathrm{CH}_{2} \mathrm{OH}(\mathbf{4 8})$ & $4-\mathrm{OH}-\mathrm{C}_{6} \mathrm{H}_{4}-\mathrm{CH}_{2} \mathrm{CH}_{2} \mathrm{OCO} \operatorname{Pr}(\mathbf{4 8 c})$ & 10 & 95 \\
\hline
\end{tabular}

${ }^{\mathrm{a}}$ Reactions were monitored by TLC. ${ }^{\mathrm{b}}$ Isolated yield. ${ }^{\mathrm{c}} \mathrm{CH}_{3} \mathrm{CN}$ was used as the solvent

Table 9. Pivaloylation ${ }^{\mathrm{a}}$ of alcohols and amines with (t-BuCO$)_{2} \mathrm{O}$ catalyzed by EDPBT

\begin{tabular}{llll}
\hline Substrate & Product & Time/ min & Yield (\%) \\
\hline $\mathrm{CH}_{3}-\left(\mathrm{CH}_{2}\right)_{8} \mathrm{CH}_{2} \mathrm{OH} \mathrm{(1)}$ & $\mathrm{CH}_{3}-\left(\mathrm{CH}_{2}\right)_{8} \mathrm{CH}_{2} \mathrm{OCOt}-\mathrm{Bu}(\mathbf{1 d})$ & 60 & 86 \\
$\mathrm{Ph}-\left(\mathrm{CH}_{2}\right)_{2} \mathrm{CH}_{2} \mathrm{OH} \mathrm{(3)}$ & $\mathrm{Ph}-\left(\mathrm{CH}_{2}\right)_{2} \mathrm{CH}_{2} \mathrm{OCOt}-\mathrm{Bu}(\mathbf{3 d})$ & 60 & 90 \\
$\mathrm{Ph}-\mathrm{CH}\left(\mathrm{CH}_{3}\right) \mathrm{OH}(\mathbf{1 6})$ & $\mathrm{Ph}-\mathrm{CH}\left(\mathrm{CH}_{3}\right) \mathrm{OCOt}-\mathrm{Bu}(\mathbf{1 6 d})$ & 90 & 80 \\
$\mathrm{PhCH}=\mathrm{CHCH}_{2} \mathrm{OH}(\mathbf{2 4})$ & $\mathrm{PhCH}=\mathrm{CHCH}_{2} \mathrm{OCOt}-\mathrm{Bu} \mathrm{(24d)}$ & 90 & 89 \\
$\mathrm{C}_{6} \mathrm{H}_{5}-\mathrm{CH}_{2}-\mathrm{NH}_{2}(\mathbf{3 9})$ & $\mathrm{C}_{6} \mathrm{H}_{5} \mathrm{CH}_{2}-\mathrm{NHCOt}^{-B u}(\mathbf{3 9 d})$ & 15 & 88 \\
$4-\mathrm{OH}-\mathrm{C}_{6} \mathrm{H}_{4}-\mathrm{CH}_{2} \mathrm{CH}_{2} \mathrm{OH}(\mathbf{4 8})$ & $4-\mathrm{OH}-\mathrm{C}_{6} \mathrm{H}_{4}-\mathrm{CH}_{2} \mathrm{CH}_{2} \mathrm{OCOt}-\mathrm{Bu}(\mathbf{4 8 d})$ & 80 & 82 \\
\hline
\end{tabular}

${ }^{\mathrm{a}}$ Reactions were monitored by TLC. ${ }^{\mathrm{b}}$ Isolated yield

Benzoic anhydride, an aromatic anhydride reacted with alcohols, amines, and thiol to give corresponding benzoates (1e, 3e, 24e, 41e, 46e, 48e and 49e). The reaction of benzoic anhydride with various alcohols, amine and thiol is summarized in Table 10. In general, the more hindered the anhydride; the slower is the acylation rate. Notably, under the present reaction condition there is not much difference in the acylation rates of alcohols, amines and thiols with acetic, propionic and isobutyric anhydride but the reaction is slower for pivalic and benzoic anhydride. This observation is consistent with the observations made by others. ${ }^{21 a, b}$ 
Table 10. Benzoylation ${ }^{\mathrm{a}}$ of alcohols, thiol and amines with $\mathrm{Bz}_{2} \mathrm{O}$ catalyzed by EDPBT

\begin{tabular}{llll}
\hline Substrate & Product & Time / h & Yield (\%) $^{\text {b }}$ \\
\hline $\mathrm{CH}_{3}\left(\mathrm{CH}_{2}\right)_{8} \mathrm{CH}_{2} \mathrm{OH}(\mathbf{1})$ & $\mathrm{CH}_{3}-\left(\mathrm{CH}_{2}\right)_{8} \mathrm{CH}_{2} \mathrm{OCOC}_{6} \mathrm{H}_{5}(\mathbf{1 e})$ & 5 & 75 \\
$\mathrm{Ph}-\left(\mathrm{CH}_{2}\right)_{2} \mathrm{CH}_{2} \mathrm{OH}(\mathbf{3})$ & $\mathrm{Ph}^{-}\left(\mathrm{CH}_{2}\right)_{2} \mathrm{CH}_{2} \mathrm{OCOC}_{6} \mathrm{H}_{5}(\mathbf{3 e})$ & 2.5 & 87 \\
$\mathrm{PhCH}=\mathrm{CHCH}_{2} \mathrm{OH} \mathrm{(24)}$ & $\mathrm{PhCH}=\mathrm{CHCH}_{2} \mathrm{OCOC}_{6} \mathrm{H}_{5}(\mathbf{2 4 e})$ & 5 & 85 \\
$\mathrm{C}_{6} \mathrm{H}_{5}-\mathrm{NH}_{2}(\mathbf{4 1})$ & $\mathrm{C}_{6} \mathrm{H}_{5}-\mathrm{NHCOC}_{6} \mathrm{H}_{5}(\mathbf{4 1 e})$ & 0.25 & 93 \\
$\mathrm{CH}_{3}\left(\mathrm{CH}_{2}\right)_{10} \mathrm{CH}_{2} \mathrm{SH} \mathrm{(46)}$ & $\mathrm{CH}_{3}\left(\mathrm{CH}_{2}\right)_{10} \mathrm{CH}_{2} \mathrm{SCOC}_{6} \mathrm{H}_{5}(\mathbf{4 6 e})$ & 5 & $68^{\mathrm{c}}$ \\
$4-\mathrm{OH}-\mathrm{C}_{6} \mathrm{H}_{4}-\mathrm{CH}_{2} \mathrm{CH} \mathrm{OH}(\mathbf{4 8})$ & $4-\mathrm{OH}-\mathrm{C}_{6} \mathrm{H}_{4}-\mathrm{CH}_{2} \mathrm{CH}_{2} \mathrm{OCOC}_{6} \mathrm{H}_{5}(\mathbf{4 8 e})$ & 2 & 80 \\
$4-\mathrm{OH}-\mathrm{C}_{6} \mathrm{H}_{4}-\mathrm{NH}_{2}(\mathbf{4 9})$ & $4-\mathrm{OH}-\mathrm{C}_{6} \mathrm{H}_{4}-\mathrm{NHCOC}_{6} \mathrm{H}_{5}(\mathbf{4 9 e})$ & 0.25 & 92 \\
\hline
\end{tabular}

${ }^{a}$ Reactions were monitored by TLC. ${ }^{b}$ Isolated yield. ${ }^{\mathrm{c}} \mathrm{CH}_{3} \mathrm{CN}$ was used as the solvent.

\section{Conclusions}

The reagent EDPBT serves as an excellent source of anhydrous $\mathrm{HBr}$ in acetone, which catalyzes acylation of structurally diverse alcohols, amines, thiols, and phenols with different anhydrides. No bromination was observed for substrates susceptible to bromination due to consumption of active bromine in EDPBT by acetone giving bromoacetone and $\mathrm{HBr}$. Solvent and steric factors in substrate as well as anhydride play a significant role during the formation of acylates. Chemoselective acylation of symmetrical diols, primary hydroxyl over secondary and phenolic, and amines over phenols has been achieved. Compared to the existing methods, which uses various acidic and basic catalysts, this method is very general, simple, gives high yield, has shorter reaction time, and is environmental friendly. In terms of compatibility and selectivity this method is superior to many of the reported methods. Due to the mild reaction conditions a number of functional groups remain intact, in spite of being capable of reacting with tribromides. The reagent EDPBT being devoid of phase transfer property and owing to the high solubility of its precursor 1,1'-(ethane-1,2-diyl)dipyridinium dibromide (EDPDB) in water, isolation of pure acylated product can be achieved only by an aqueous work-up circumventing the need of chromatographic purification. Further, the superiority of the process lies in the recyclability of the reagent. The extensive waste of EDPBT reagent is avoided by regeneration of the reagent from the aqueous layer. It can be used without any loss of its activity for further transformations which is of significant interest.

\section{Experimental Section}

General Procedures. All the reagents were commercial grade and purified according to the established procedures. Organic extracts were dried over anhydrous sodium sulfate. Solvents were removed in a rotary evaporator under reduced pressure. Silica gel (60-120 mesh size) was 
used for the column chromatography. Reactions were monitored by TLC on silica gel $60 \mathrm{~F}_{254}$ $(0.25 \mathrm{~mm})$. NMR spectra were recorded in $\mathrm{CDCl}_{3}$ or DMSO- $\mathrm{d}_{6}$ with tetra methyl silane as the internal standard for ${ }^{1} \mathrm{H}$ NMR $\left(300\right.$ and $400 \mathrm{MHz}$ ) and $\mathrm{CDCl}_{3}$ or DMSO- $\mathrm{d}_{6}$ solvents as internal standard for ${ }^{13} \mathrm{C}$ NMR (100 MHz). IR spectra were recorded in $\mathrm{KBr}$ or neat. GC-MS were recorded using a capillary column $(30 \times 0.25 \mathrm{~mm}$ X $0.25 \mathrm{~m} \mu)$ in EI mode. The following acylates derived from the parent alcohols, amines and phenols by reacting with different anhydrides have been reported in the literature: acetates $\mathbf{1 a},{ }^{23 a, b} \mathbf{2 a},{ }^{8 f} \mathbf{3 a},{ }^{19 a, 20 a, 42 a, b} \mathbf{4 - 5 a},{ }^{21 e} \mathbf{7 - 8 a},{ }^{8 f}$ 9a, ${ }^{21 \mathrm{~d}} \mathbf{1 0 a},{ }^{20 \mathrm{~b}} \mathbf{1 1 a},{ }^{8 \mathrm{f}} \mathbf{1 2 a},{ }^{15 \mathrm{~b}} \mathbf{1 4 a},{ }^{21 \mathrm{e}} \mathbf{1 5 a},{ }^{21 \mathrm{~b}, 42 \mathrm{~b}} \mathbf{1 6 a},{ }^{20,21 \mathrm{~b}, 23 \mathrm{~b}} \mathbf{1 7 a},{ }^{8 \mathrm{f}} \mathbf{1 9 a},{ }^{52 \mathrm{~d}} \mathbf{1 9 a},{ }^{20 \mathrm{a}, 8 \mathrm{f}} \mathbf{2 1 a},{ }^{18 \mathrm{k}} \mathbf{2 1}$ 'a,${ }^{51 \mathrm{e}}$ 22a $,{ }^{18 \mathrm{j}} \mathbf{2 3 a},{ }^{20,21 \mathrm{~b}} \mathbf{2 4 a},{ }^{20 \mathrm{a}, 21 \mathrm{e}, 23 \mathrm{~b}, 42 \mathrm{a}-\mathrm{b}, 43} \mathbf{2 5 a},{ }^{8 \mathrm{f}} \mathbf{2 7 a},{ }^{18 \mathrm{e}, 20 \mathrm{a}} \mathbf{2 8 a},{ }^{52 \mathrm{a}} \mathbf{2 9 a},{ }^{52 \mathrm{e}, 8 \mathrm{f}} \mathbf{3 0 a},{ }^{8 \mathrm{f}} \mathbf{3 1 a},{ }^{52} \mathbf{3 2 a},{ }^{21 \mathrm{e}} \mathbf{3 3 a},{ }^{49}$

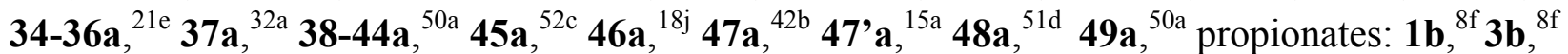
$16 b,{ }^{8 \mathrm{f}} \mathbf{2 4 b},{ }^{8 \mathrm{f}} 41 \mathrm{~b},{ }^{50 \mathrm{a}} 48 \mathrm{~b},{ }^{8 \mathrm{f}} 49 \mathrm{~b},{ }^{50 \mathrm{a}}$ isobutyrate: $1 \mathbf{c},{ }^{8 \mathrm{f}} \mathbf{3 c},{ }^{8 \mathrm{f}} \mathbf{1 6 c},{ }^{8 \mathrm{f}} \mathbf{4 1 c},{ }^{5 \mathrm{f}} \mathbf{4 8 c},{ }^{8 \mathrm{f}}$ pivalates: $1 \mathbf{d},{ }^{8 \mathrm{f}} \mathbf{3 d},{ }^{8 \mathrm{f}}$ 16d, ${ }^{8 f, 21 b} \mathbf{2 4 d},{ }^{43} 39 d{ }^{43} 48 d$, ${ }^{8 f}$ benzoates: 1 e, ${ }^{52 b}, 3 e,{ }^{42 a}, 24 e,{ }^{43,21 e} 41 e,{ }^{50 a} 49 e^{50 a}$

General procedure for reaction of alcohols, amines, and phenols with acetic, propionic, isobutyric, and pivalic anhydride. Reagent EDPBT $(0.25 \mathrm{mmol}, 166.5 \mathrm{mg})$ was added to a stirred solution of acetone $(10 \mathrm{~mL})$ followed by 3-phenyl propanol $3(688 \mu \mathrm{L}, 5 \mathrm{mmol})$ and acetic anhydride $(590 \mu \mathrm{L}, 6.25 \mathrm{mmol})$. The progress of reaction was monitored by TLC. After completion of the reaction, solvent was evaporated in a rotary evaporator and admixed with ethyl acetate $(25 \mathrm{~mL})$. Organic layer was washed successively with water $(2 \times 5 \mathrm{~mL})$ followed by saturated $\mathrm{NaHCO}_{3}$ solution $(5 \mathrm{~mL})$. Organic layer was dried over anhydrous $\mathrm{Na}_{2} \mathrm{SO}_{4}$, filtered and solvent was concentrated in a rotary evaporator. The compound was sufficiently pure but for analytical data it was purified by passing it over a short column of silica gel, using a mixture of hexane and ethyl acetate as eluent to yield $855 \mathrm{mg}(95 \%)$ of acetylated product 3a. The aqueous layer containing EDPDB was retained for the regeneration of EDPBT. 9

General procedure for benzoylation of alcohols, amines and phenols with benzoic anhydride. Similar to the acetylation with acetic anhydride except $5 \mathrm{mmol}$ of benzoic anhydride was used per $5 \mathrm{mmol}$ of the substrate.

\section{General procedure for the reaction of thiol with acetic, propionic, and isobutyric}

anhydride. Similar to the reaction of alcohols with anhydrides, but acetonitrile was used instead of acetone as the reaction medium.

General procedure for benzoylation of thiols with benzoic anhydride. Similar to the acetylation with acetic anhydride but $5 \mathrm{mmol}$ of benzoic anhydride was used per $5 \mathrm{mmol}$ of the substrate in acetonitrile.

General procedure for selective monoacetylation of diols. Similar to the acetylation of alcohols with acetic anhydride except $6 \mathrm{mmol}$ of acetic anhydride was used per $5 \mathrm{mmol}$ of the substrate with lot wise addition of anhydride over a period of 30 minutes. The products were purified over a column of silica gel, using a mixture of hexane and ethyl acetate as eluent.

Regeneration of 1,1'-(ethane-1,2-diyl)dipyridinium bistribromide (EDPBT). To the aqueous layer originating from the above reaction containing 1,1'-(ethane-1,2-diyl)dipyridinium dibromide (EDPDB) ( 1 equiv) and approximately 0.66 equiv of bromide ion (some of the bromine being consumed by the bromination of acetone) was concentrated to $5 \mathrm{~mL}$, extracted 
with ethylacetate $(10 \mathrm{~mL})$ to get rid of organic contaminants. To the aqueous layer was added $\mathrm{KBr}$ (354mg, 3 equiv) followed by pinchwise addition of Oxone ${ }^{\circledR}$ (1.228g, 2 equiv) under stirring. The precipitated orange solid was filtered to yield $1.17 \mathrm{~g}(88 \%)$ of the bis-tribromide. The recovered reagent is identical in all respect to the parent EDPBT reagent.

In principle aqueous layers of several reactions were combined and kept for several days to allow the water to evaporate and proportionate amounts of $\mathrm{KBr}$ and Oxone ${ }^{\circledR}$ were added to regenerate the reagent.

\section{Acknowledgements}

B. K. P and S. N acknowledge the support of this research from CSIR New Delhi 01(1946)/04 and V.K and R.G acknowledges the financial support to the Institute. Thanks are due to CIF IIT Guwahati and RSIC, Lucknow for providing NMR spectra.

\section{References}

1. (a) Bora, U.; Bose, G.; Chaudhuri, M. K.; Dhar, S. S.; Gopinath, R.; Khan, A. T.; Patel, B. K. Org. Lett. 2000, 2, 247. (b) Chaudhuri, M. K.; Khan, A. T.; Patel, B. K.; Dey, D.; Kharmawophlang, W.; Lakshmiprabha, T. R.; Mandal, G. C. Tetrahedron Lett. 1998, 39, 8163. (c) Paquet, L.A., Ed. Encyclopedia of Reagent for Organic Synthesis; Wiley: New York, 1995, Vol. 12; p 4738.

2. Avramoff, M.; Weiss, J.; Schächter, O. J. Org. Chem. 1963, 23, 3256.

3. Chaudhuri, M. K.; Bora, U.; Dehury, S. K.; Dey, D.; Dhar, S. S.; Kharmawophlang, W.; Choudary, B. M.; Mennepalli, L. K. US Patent 2004, 126308.

4. (a) Kajigaeshi, S.; Kakinami, T.; Tokiyama, H.; Hirakawa, T.; Okamoto, T. Chem. Lett. 1987, 627. (b) Jordan, A. D.; Luo, C.; Reitz, A. B. J. Org. Chem. 2003, 68, 8693. (c) Kajigaeshi, S.; Kakinami, T.; Tokiyama, H.; Yamasaki, H.; Hirakawa, T.; Okamoto, T. Bull. Chem. Soc. Jpn. 1987, 60, 2667.

5. (a) Salazar, J.; Dorta, R. Synlett 2004, 1318. (b) Tanaka, K.; Shiraishi, R.; Toda, F. J. Chem. Soc., Perkin Trans. 1, 1999, 3069.

6. (a) Markovic, R.; Baranac, M.; Dzambaski, Z. Heterocycles, 2004, 63, 851. (b) Fischer, L. F.; Fischer, M. Reagents for Organic Synthesis. Wiley, New York, 1967, p. 967. (c) Reeves, W. P.; Lu, C. V.; Schulmeier, B.; Jonas, L.; Hatlevik, O. Synth. Commun. 1998, 28, 499. (d) Paquet, L. A. (ed) Encyclopedia of Reagent for Organic Synthesis; Wiley: New York: 1995; Vol 6, p. 4370.

7. Muathen H. A. J. Org. Chem. 1992, 57, 2740.

8. (a) Gopinath, R.; Patel, B. K. Org. Lett. 2000, 2, 4177. (b) Naik, S.; Gopinath, R.; Patel, B. K. Tetrahedron Lett. 2001, 42, 7679. (c) Gopinath, R.; Haque, Sk. J.; Patel, B. K. J. Org. Chem. 
2002, 67, 5842. (d) Naik, S.; Gopinath, R.; Goswami, M.; Patel, B. K. Org. Biomol. Chem. 2004, 1670. (e) Kavala, V.; Patel, B. K. Eur. J. Org. Chem. 2005, 441. (f) Naik, S.; Kavala. V.; Gopinath, R.; Patel, B. K. Arkivoc 2006, (i), 119.

9. Kavala, V.; Naik, S.; Patel, B. K. J. Org. Chem. 2005, 70, 4267.

10. Greene, T. W.; Wuts, P. G. M. Protective Groups in Organic Synthesis, $3^{\text {rd }}$ ed.; John Wiley and Sons: New York, 1999.

11.(a) Rowan, S. J.; Cantrill, S. J.; Cousins, G. R. L.; Sanders, J. K. M.; Stoddart J. F. Angew Chem., Int. Ed. 2002, 41, 898. (b) Karan, C.; Miller, B. L. Drug Discovery Today 2000, 5 , 67.

12.Larock, R. C. In Comprehensive Organic Transformations, VCH: New York, 1989; p 980.

13. Schlubach, H. H.; Repenning, K. Angew Chem., 1959, 71, 193.

14.(a) Steglich, W.; Höfle, G. Angew. Chem. Int. Ed. Engl. 1969, 8, 981. (b) Höfle, G.; Steglich, W.; Vorbrüggen, H. Angew. Chem. Int. Ed. Engl. 1978, 17, 569. (c) Scriven, E. F.V. Chem. Soc. Rev. 1983, 12, 129. (d) Berry, D. J.; DiGiovanna, C. V.; Metrick, S. S.; Murugan, R. Arkivoc 2001(ii) 944.

15.(a) Orita, A.; Sakamato, K.; Hamada, Y.; Mitsutome, A.; Otera, J. Tetrahedron 1999, 55, 2899. (b) Orita, A.; Ito, T.; Yasui, Y.; Otera, J. Synlett 1999, 1927.

16.(a) Vedejs, E.; Bennett, N. S.; Conn, L. M.; Diver, S. T.; Gingras, M.; Lin, S.; Oliver, P. A.; Peterson, M. J. J. Org. Chem. 1993, 58, 7286. (b) Vedejs, E.; Diver, S. T. J. Am. Chem. Soc. 1993, 115, 3358. (c) Buckler, S. A. J. Am . Chem. Soc. 1962, 84, 3093.

17.Cope, A. C.; Herrick, E. C. Organic Syntheses, Collect. Vol. 4, Wiley: New York: 1963; p.304.

18.(a) Backer, R. H.; Bordwell, F. G. Org. Synth. 1955, 3, 141. (b) Iqbal, J.; Srivastava, R. R. J. Org. Chem. 1992, 57, 2001. (c) Chandrasekhar, S.; Ramachander, T.; Takhi, M. Tetrahedron Lett. 1998, 39, 3263. (d) Vedejs, E.; Daugulis, O. J. Org. Chem. 1996, 61, 5702. (e) De, S. K. Tetrahedron Lett. 2004, 45, 2919. (f) Chakraborti, A. K; Gulhane, R. Tetrahedron Lett. 2003, 44, 6749. (g) Chakraborti, A. K; Gulhane, R Synlett 2004, 627. (h) Phukan, P. Tetrahedron Lett. 2004, 450, 4785. (i) Karimi, B.; Seradj, H. Synlett 2001, 519. (j) Khan, A. T.; Chaudhury, L. H. Ghosh, S. Eur. J. Org. Chem. 2005, 2782. (k) Sarvari, H.; Hashem, M; S.; Tetrahedron 2005, 61, 10903.

19.(a) Procopiou, P. A.; Baugh, S. P. D.; Flack, S. S.; Inglis, G. G. A. J. Org. Chem. 1998, 63, 2342. (b) Procopiou, P. A.; Baugh, S. P. D.; Flack, S. S.; Inglis, G. G. A. Chem. Commun. 1996, 2625.

20. (a) Karimi, B.; Maleki, J. J. Org. Chem. 2003, 68, 4951. (b) Chauhan, K. K.; Frost, C. G.; Love, I.; Waite, D. Synlett 1999, 1743. (c) Mukaiyama, T.; Shiina, I.; Miyashra, M. Chem. Lett. 1992, 625.

21.(a) Orita, A.; Tanahashi, C.; Kakuda, A.; Otera, J. Angew. Chem. Int. Ed. 2000, 39, 2877. (b) Orita, A.; Tanahashi, C.; Kakuda, A.; Otera, J. J. Org. Chem. 2001, 66, 8926.(c) Yadav, J. S.; Reddy, B. V. S.; Swamy, T.; Rao, K. R. Tetrahedron Lett. 2004, 45, 6037. (d) Carrigan, M. D.; Freiberg, D. A.; Smith, R. C.; Zerth, H. M.; Mohan, R. S. Synthesis 2001, 2091. (e) 
Mohammadpoor-Baltork, I.; Aliyan, H.; Khosropour, A. R. Tetrahedron 2001, 57, 5851. (f) Peterson, K. E.; Smith, R. C.; Mohan, R. S. Tetrahedron Lett. 2003, 44, 7723. (g) Leonard, N. M.; Wieland, L. C.; Mohan, R. S. Tetrahedron 2002, 58, 8373. (h) Gaspard-Iloughmane, H.; Roux, C. L. Eur. J. Org. Chem. 2004, 2517.

22.Izumi, J.; Shiina, I.; Mukaiyama, T. Chem Lett. 1995, 141.

23. (a) Saravanan, P.; Singh, V. K. Tetrahedron Lett. 1999, 40, 2611. (b) Chandra, K. L.;

Saravanan, P.; Singh, R. K.; Singh, V. K. Tetrahedron 2002, 58, 1369.

24. (a) Ishihara, K.; Kubota, M.; Kurihara, H.; Yamamoto, H. J. Am. Chem. Soc. 1995, 117 , 4413. (b) Ishihara, K.; Kubota, M.; Kurihara, H.; Yamamoto, H. J. Org. Chem. 1996, 61, 4560. (c) Zhou, H.; Pendri, A.; Greenwald, R. B. J. Org. Chem. 1998, 63, 7559.(d)

Jarowicki, K.; Kocienski, P. Org. Synth. 1997, 454.

25.Barrett, A. G. M.; Braddock, D. C. Chem. Commun. 1997, 351.

26.Damen, E. W. P.; Braamer, L.; Scheeren, H. W. Tetrahedron Lett. 1998, 39, 6081.

27.Clarke, P. A.; Kayaleh, N. E.; Smith, M. A.; Baker, J. R.; Bird, S. J.; Chan, C. J. Org. Chem. 2002, 67, 5226.

28.Dalpozzo, R.; Nino, A. D.; Maiuolo, L.; Procopio, A.; Nardi, M.; Bartoli, G.; Romeo, R. Tetrahedron Lett. 2003, 44, 5621.

29.Alleti, R.; Perambuduru, M.; Samantha, S.; Reddy, V. P. J. Mol. Cat. A: Chemical, 2005, $226,57$.

30.Miyashita, M.; Shiina, I.; Miyoshi, S.; Mukaiyama, T. Bull. Chem. Soc. Jpn. 1993, 66, 1516.

31.(a) Nakae, Y.; Kusaki, I.; Sato, T. Synlett 2001, 1584. (b) Bartoli, G.; Bosco, M.; Marcantoni, E.; Massaccesi, M.; Rinaldi, S.; Sambri, L. Tetrahedron Lett. 2002, 43, 6331.

32.(a) Chakraborti, A. K.; Sharma, L.; Gulhane, R. S. Tetrahedron 2003, 59, 7661. (b) Bartoli, G.; Bosco, M.; Dalpozzo, R.; Marcantoni, E.; Massaccesi, M.; Rinaldi, S.; Sambri, L. Synlett 2003, 39.

33. Chakraborti, A. K.; Gulhane, R.; Shivani. Synlett 2003, 1805.

34.Kumareswaran, R.; Gupta, A.; Vankar, Y. D. Synth. Commun. 1997, 27, 277.

35. Chakraborti, A. K.; Gulhane, R. Chem. Commun. 2003, 1896.

36.Ishihara, K.; Kubota, M.; Yamamoto, H. Synlett 1996, 265.

37.Kumareswaran, R.; Pachamuthu, K.; Vankar, Y. D. Synlett 2000, 1652.

38.Kumar, P.; Pandey, R. K.; Bodas, M. S.; Dongare, M. K. Synlett 2001, 206.

39. Orita, A.; Mitsutome, A.; Otera, J. J. Org. Chem. 1998, 63, 2420.

40.Firouzabadi, H.; Iranpoor, N.; Nowrouzi, F.; Amani, K. Chem.Commun. 2003, 764.

41. Sharghi, H.; Sarvari, M. H. Tetrahedron 2003, 59, 3627.

42.(a) Yadav, V. K.; Babu, K. G. J. Org. Chem. 2004, 69, 577. (b) Yadav, V. K.; Babu, K. G.; Mittal, M. Tetrahedron 2001, 57, 7047.

43.Chen, C. -T.; Kuo, J.-H.; Pawar, V. D.; Munot, Y. S.; Weng, S.-S.; Ku, C. -H.; Liu, C. -Y. J. Org. Chem. 2005, 70, 1188. 
44.Chen, C. -T.; Kuo, J. -H.; Li, C. -H.; Barhate, N. B.; Hon, S. -W.; Li, T. -W.; Chao, S. -D.; Liu, C. -C.; Li, Y. -C.; Chang, I-H.;Lin, J. -S.; Liu, C. -J.; Chou, Y-C. Org. Lett. 2001, 3, 3729.

45.Olah, G. A.; Prakash, G. K. S. Super Acids; Wiley: New York, 1985.

46. Schumacher, J. C. Perchlorates-Their Properties, Manufacture and Uses; ACS Monograph Series, Reinhold: New York 1960.

47.Long, J. Chemical health and Safety 2002, 9, 12.

48. (a) Humphrey, C. E.; Easson, M. A. M.; Tierney, J. P.; Turner, N. J. Org. Lett. 2003, 5, 849.

(b) Atkinson, R. S.; Barker, E.; Sutcliffe, M. J. Chem. Commun. 1996, 1051. (c) Ahmed, S.; Iqbal, J. J. Chem. Soc. Chem. Commun. 1987, 114. (d) D’Sa, B. A.; Verkade, J. G. J. Org. Chem. 1996, 61, 2963. (e) Katritzky, A. R.; He, H. -Y.; Suzuki, K. J. Org. Chem. 2000, 65, 8210 .

49.Li, J- S.; Li, A. -X. J. Chem. Soc. Perkin Trans I, 1998, 1913.

50.(a) Naik, S.; Bhattacharjya, G.; Talukdar, B.; Patel, B. K. Eur. J. Org. Chem. 2004, 1254. (b) Ranu, B. C.; Dey, S. S.; Hajra, A. Green Chemistry, 2003, 5, 44.

51.(a) Framis, V.; Camps, F.; Clapés, P. Tetrahedron Lett. 2004, 45, 5031. (b) Clarke, P. A. Tetrahedron Lett. 2002, 43, 4761. (c) Nishiguchi, T.; Kawamine, K.; Ohtsuka, T. J. Org. Chem. 1992, 57, 312. (d) Breton, G. W. J. Org. Chem. 1997, 62, 8952. (e) Srinivas, K. V. N. S.; Mahender, I.; Das, B. Synlett 2003, 2419.

52.(a) Reddy, C. S.; Smitha, G.; Chandrasekhar, S. Tetrahedron Lett. 2003, 44, 4693.(b)

Velusamy, S.; Borpuzari, S.; Punniyamurthy, T. Tetrahedron 2005, 61, 2011.(c) Heyde, C.; Zug, I.; Hartmann, H. Eur. J. Org. Chem.2000, 3273. (d) Choi, J, H.; Kim, Y. H.; Nam, S. H.; Shin, S. T.; Kim, M. -J; Park, J. Angew Chem., Int. Ed. 2002, 41, 2373. (e) Wu, Z.; Stanley, R, R.; Pittman, C. U. J. Org. Chem.1999, 64, 8386. (f) Humphrey, C. E.; Easson, M. A. M.; Tierney, J. P.; Turner, N. J. Organic Lett. 2003, 5, 849.

53.Spectroscopic data of compounds, 6a. ${ }^{1} \mathrm{H}$ NMR $\left(400 \mathrm{MHz}, \mathrm{CDCl}_{3}\right) \delta 2.20(\mathrm{~s}, 3 \mathrm{H}), 5.26(\mathrm{~s}$, $2 \mathrm{H}), 7.56(\mathrm{~d}, 1 \mathrm{H}, J=8.8 \mathrm{~Hz}), 8.13\left(\mathrm{dd}, 1 \mathrm{H}, J_{l}=8.4 \mathrm{~Hz}, J_{2}=2.8 \mathrm{~Hz}\right), 8.29(\mathrm{~d}, 1 \mathrm{H}, J=2.8$ $\mathrm{Hz}) ;{ }^{13} \mathrm{C} \mathrm{NMR}\left(100 \mathrm{MHz}, \mathrm{CDCl}_{3}\right) \delta .21 .2,62.8,124.1,124.2,130.6,135.9,139.9,146.8$, 170.4; Mass $(\mathrm{m} / \mathrm{z})$ 229. 13a: ${ }^{1} \mathrm{H}$ NMR $\left(400 \mathrm{MHz} \mathrm{CDCl}_{3}\right) \delta 2.07(\mathrm{~s}, 12 \mathrm{H}), 4.12(\mathrm{~s}, 8 \mathrm{H}) .{ }^{13} \mathrm{C}$ NMR $\left(100 \mathrm{MHz}, \mathrm{CDCl}_{3}\right) \delta 21.1,41.9,62.6,170.5$; Mass $(\mathrm{m} / z)$ 304. 13'a: ${ }^{1} \mathrm{H}$ NMR (400 $\left.\mathrm{MHz} \mathrm{CDCl}_{3}\right) \delta 1.47(\mathrm{~s}, 6 \mathrm{H}), 2.06(\mathrm{~s}, 6 \mathrm{H}), 3.74(\mathrm{~s}, 4 \mathrm{H}), 4.10(\mathrm{~s}, 4 \mathrm{H}) ;{ }^{13} \mathrm{C} \mathrm{NMR}(100 \mathrm{MHz}$, $\left.\mathrm{CDCl}_{3}\right) \delta$ 21.2, 23.9, 37.4, 62.2, 63.5, 98.8, 170.8; Mass $(\mathrm{m} / \mathrm{z})$ 260. 13"a: ${ }^{1} \mathrm{H}$ NMR (400 $\left.\mathrm{MHz} \mathrm{CDCl}_{3}\right) \delta 1.40(\mathrm{~s}, 12 \mathrm{H}), 3.72(\mathrm{~s}, 8 \mathrm{H}) ;{ }^{13} \mathrm{C} \mathrm{NMR}\left(100 \mathrm{MHz}, \mathrm{CDCl}_{3}\right) \delta 24.0,33.0,64.4$, 98.8; Mass $(m / z)$ 216; Anal. Calcd for $\mathrm{C}_{11} \mathrm{H}_{20} \mathrm{O}_{4}$ : C, 61.09; H, 9.32. Found C, 61.25; H, 9.26. 18a: ${ }^{1} \mathrm{H}$ NMR $\left(300 \mathrm{MHz}, \mathrm{CDCl}_{3}\right) \delta 1.51(\mathrm{~d}, 3 \mathrm{H}, J=6.6 \mathrm{~Hz}), 2.06(\mathrm{~s}, 3 \mathrm{H}), 5.83(\mathrm{q}, 1 \mathrm{H}, J=$ $6.6 \mathrm{~Hz}), 7.30$ (m, 4H); ${ }^{13} \mathrm{C} \mathrm{NMR}\left(75 \mathrm{MHz}, \mathrm{CDCl}_{3}\right) \delta .21 .2,22.1,71.5,127.5,128.6,133.5$, 140.2, 170.2; Mass (m/z) 198; Anal. Calcd for $\mathrm{C}_{10} \mathrm{H}_{11} \mathrm{ClO}_{2}$ : C, 60.46; $\mathrm{H}, 16.11$. Found $\mathrm{C}$, 60.58; H, 15.95. 20a: ${ }^{1} \mathrm{H}$ NMR (400 MHz, $\left.\mathrm{CDCl}_{3}\right) \delta 2.13(\mathrm{~s}, 3 \mathrm{H}), 6.74(\mathrm{~s}, 1 \mathrm{H}), 7.24(\mathrm{t}, 2 \mathrm{H}, J$ $=7.5 \mathrm{~Hz}), 7.35(\mathrm{t}, 2 \mathrm{H}, J=7.3 \mathrm{~Hz}), 7.50(\mathrm{~d}, 2 \mathrm{H}, J=7.5 \mathrm{~Hz}), 7.60(\mathrm{~d}, 2 \mathrm{H}, J=7.5 \mathrm{~Hz}) ;{ }^{13} \mathrm{C}$ NMR $\left(100 \mathrm{MHz}, \mathrm{CDCl}_{3}\right) \delta$. 21.1, 75.0, 119.9, 125.8, 127.7, 129.4, 140.9, 141.9, 171.7; Mass 
$(m / z)$ 224; Anal. Calcd for $\mathrm{C}_{15} \mathrm{H}_{12} \mathrm{O}_{2}$ : C, 80.34; H, 5.39. Found $\mathrm{C}, 80.59 ; \mathrm{H}, 5.21 .26 \mathrm{a}:{ }^{1} \mathrm{H}$ NMR (400 MHz, $\left.\mathrm{CDCl}_{3}\right) \delta 1.25(\mathrm{~s}, 3 \mathrm{H}), 1.43(\mathrm{~s}, 9 \mathrm{H}), 2.10(\mathrm{~s}, 3 \mathrm{H}), 3.61$ (q, 2H, $J=11.2$ $\mathrm{Hz}), 4.21(\mathrm{~m}, 2 \mathrm{H}), 3.57$ (brs, $1 \mathrm{H}) .{ }^{13} \mathrm{C}$ NMR $\left(100 \mathrm{MHz}, \mathrm{CDCl}_{3}\right) \delta 20.4,21.3,28.7,56.5$, 66.6, 67.2, 80.4, 155.8, 171.3; Mass $(\mathrm{m} / \mathrm{z})$ 247. 46b: ${ }^{1} \mathrm{H}$ NMR (400 MHz, $\left.\mathrm{CDCl}_{3}\right) \delta 0.88(\mathrm{t}$, $3 \mathrm{H}, J=7.0 \mathrm{~Hz}), 1.17(\mathrm{t}, 3 \mathrm{H}, J=7.6 \mathrm{~Hz}), 1.25(\mathrm{brs}, 18 \mathrm{H}), 1.58(\mathrm{~m}, 2 \mathrm{H}), 2.56(\mathrm{q}, 2 \mathrm{H}, J=7.6$ $\mathrm{Hz}), 2.86(\mathrm{t}, 2 \mathrm{H}, J=7.6 \mathrm{~Hz}) .{ }^{13} \mathrm{C} \mathrm{NMR}\left(100 \mathrm{MHz}, \mathrm{CDCl}_{3}\right) \delta 10.2,14.6,23.1,29.2,29.3$, 29.5, 29.8, 29.9, 30.0, 30.02, 30.05, 32.3, 37.8; Mass $(\mathrm{m} / z) 258$; Anal. Calcd for $\mathrm{C}_{15} \mathrm{H}_{30} \mathrm{OS}$ : C, 69.71; H, 11.70; S, 12.41. Found C, 69.88; H, 11.62; S, 12.13. 24c: ${ }^{1} \mathrm{H}$ NMR (400 MHz, $\left.\mathrm{CDCl}_{3}\right) \delta 1.20(\mathrm{~d}, 6 \mathrm{H}, J=7.2 \mathrm{~Hz}), 2.60(\mathrm{~m}, 1 \mathrm{H}), 4.73\left(\mathrm{dd}, 2 \mathrm{H}, J_{l}=6.4 \mathrm{~Hz}, J_{2}=1.6 \mathrm{~Hz}\right)$, $6.29(\mathrm{~m}, 1 \mathrm{H}), 6.65(\mathrm{~d}, 1 \mathrm{H}, J=16 \mathrm{~Hz}), 7.25(\mathrm{~m}, 1 \mathrm{H}), 7.33(\mathrm{~m}, 2 \mathrm{H}), 7.39(\mathrm{~m}, 2 \mathrm{H}) .{ }^{13} \mathrm{C}$ NMR $\left(100 \mathrm{MHz}, \mathrm{CDCl}_{3}\right) \delta .19 .5,34.5,65.2,123.6,126.8,128.2,128.8,134.1,136.4,177.0$; Mass $(\mathrm{m} / z)$ 204; Anal. Calcd for $\mathrm{C}_{13} \mathrm{H}_{16} \mathrm{O}_{2}$ : C, 76.44; H, 7.90. Found $\mathrm{C}, 76.58 ; \mathrm{H}, 7.78 .46 \mathrm{c}:{ }^{1} \mathrm{H}$ NMR (400 MHz, $\left.\mathrm{CDCl}_{3}\right) \delta 0.88(\mathrm{t}, 3 \mathrm{H}, J=6.8 \mathrm{~Hz}), 1.18$ (d, $\left.6 \mathrm{H}, J=7.2 \mathrm{~Hz}\right), 1.26$ (brs, $18 \mathrm{H}$ ), $1.55(\mathrm{~m}, 2 \mathrm{H}), 2.72$ (septet, $1 \mathrm{H}, J=7.2 \mathrm{~Hz}), 2.84$ (t, $2 \mathrm{H}, J=7.2 \mathrm{~Hz}) .{ }^{13} \mathrm{C}$ NMR $(100 \mathrm{MHz}$, $\left.\mathrm{CDCl}_{3}\right) \delta 14.6,19.8,23.1,28.9,29.3,29.5,29.8,29.9,30.0,30.1,32.3,43.5,204.3$; Mass $(\mathrm{m} / \mathrm{z})$ 272; Anal. Calcd for $\mathrm{C}_{16} \mathrm{H}_{32} \mathrm{OS}$ : C, 70.53; H, 11.84; S,11.77. Found C, 71.03; H, 11.69; S, 11.69. 46e: ${ }^{1} \mathrm{H}$ NMR (400 MHz, $\left.\mathrm{CDCl}_{3}\right) \delta 0.88$ (t, $\left.3 \mathrm{H}, J=7.2 \mathrm{~Hz}\right), 1.26(\mathrm{~s}, 18 \mathrm{H})$, 1.67 (m, 2H), 3.06 (t, 2H, $J=7.6 \mathrm{~Hz}), 7.44$ (t, $2 \mathrm{H}, J=7.6 \mathrm{~Hz}), 7.54$ (t, $1 \mathrm{H}, J=8.0 \mathrm{~Hz}), 7.96$ $(\mathrm{d}, 2 \mathrm{H}, J=8.0 \mathrm{~Hz}) .{ }^{13} \mathrm{C} \mathrm{NMR}\left(100 \mathrm{MHz}, \mathrm{CDCl}_{3}\right) \delta 14.2,22.8,29.0,29.2,29.3,29.4,29.60$, 29.66, 29.72, 32.0, 127.0, 128.4, 133.0, 137.1, 191.8; Mass $(\mathrm{m} / \mathrm{z})$ 306; Anal. Calcd for $\mathrm{C}_{19} \mathrm{H}_{30}$ OS: C, 74.45; H, 9.87; S,10.46. Found C, 73.98; H, 9.93; S, 10.22. 48e: ${ }^{1} \mathrm{H}$ NMR $\left(400 \mathrm{MHz}, \mathrm{CDCl}_{3}\right) \delta 3.00$ (t, 2H, $\left.J=6.8 \mathrm{~Hz}\right), 4.48$ (t, 2H, $J=6.8 \mathrm{~Hz}$ ), 5.00 (brs, $\left.1 \mathrm{H}\right), 6.78$ $(\mathrm{d}, 2 \mathrm{H}, J=8.0 \mathrm{~Hz}), 7.14(\mathrm{~d}, 2 \mathrm{H}, J=8.0 \mathrm{~Hz}), 7.42(\mathrm{t}, 2 \mathrm{H}, J=7.6 \mathrm{~Hz}), 7.55(\mathrm{~m}, 1 \mathrm{H}), 8.00(\mathrm{~d}$, $2 \mathrm{H}, J=7.6 \mathrm{~Hz}) .{ }^{13} \mathrm{C} \mathrm{NMR}\left(100 \mathrm{MHz} \mathrm{CDCl}_{3}\right) \delta 34.7,66.2,115.6,128.6,129.8,129.9,130.2$, 130.3, 133.2, 154.6, 166.9. Mass $(\mathrm{m} / \mathrm{z}) 242$. 